\title{
Adaptive uplink scheduling model for WiMAX network using evolutionary computing model
}

\author{
Mahesh D S ${ }^{1}$, Chandramouli $\mathbf{H}^{2}$, Sanjay R Chitnis ${ }^{3}$ \\ ${ }^{1}$ Department of CSE, Faculty of Engineering, CHRIST (Deemed to be University), India \\ ${ }^{2}$ Department of CSE, East Point College of Engineering, India \\ ${ }^{3}$ Department of CSE, Dayananda Sagar University, India
}

\begin{tabular}{l} 
Article Info \\
\hline Article history: \\
Received Sep 9, 2018 \\
Revised Nov 17, 2018 \\
Accepted Feb 22, 2019 \\
\hline Keywords: \\
Evolutionary computing \\
Genetic algorithm \\
Modulation \\
Multi-objective \\
WiMAX
\end{tabular}

Article Info

\begin{abstract}
The increased usage of smart phones has led to increase usage an internet based application services. These application requires different quality of service (QoS) and bandwidth requirement. WiMAX is an efficient network to provision high bandwidth connectivity and coverage to end user. To meet QoS requirement the exiting model used adaptive model selection scheme. However, these model induce bandwidth wastage as it does not considers any feedback information for scheduling. This work present an Adaptive Uplink Scheduling (AUS) by optimizing MAC layer using Multi-Objective Genetic Algorithm (MOGA). The MAC scheduler use feedback information from both physical layer and application layer. Further, to meet QoS requirement of application and utilize bandwidth efficiently this paper presented an adaptive modulation selection scheme based on user application requirement using MOGA. Our model provides application level based QoS provisioning for WiMAX network. Experiment are conducted to evaluate performance of AUS over exiting model. The overall result attained shows AUS model attain good performance in term of throughput, successful packet transmission and packet collision.
\end{abstract}

Copyright $@ 2019$ Institute of Advanced Engineering and Science. All rights reserved.

\section{Corresponding Author:}

Mahesh D S,

Department of CSE, Faculty of Engineering,

CHRIST (Deemed to be University),

Bangalore, India.

Email: mahesh.ds720@rediffmail.com

\section{INTRODUCTION}

In the rural area, the battle field and remote area, the unfeasibility and economically possess a big challenges because of wired network configuration to access. Thus worldwide interoperability for microwave access (WI-MAX) [1] that provides best result for long and wide range transmission in wireless communication. Wi-MAX offers customary wireless access with pre-defined QoS and faster transmission rate. Wi-MAX is designed to provision services to IEEE 802.11 standard that gives mobile user access to facility. The QoS becomes a key problem [2], due to increases popularity and adoption of wireless network, and thus the Wi-MAX provides superior and fine-grained service than the other technology. The Wi-MAX services are categorized in to real-time services such as Unsolicited Grant Service (UGS): This services support the constant bit rate traffic like voice based application services. Polling service such as Real time polling services (rtPS): This services support flexible size of data packets which is composed of constantinformation streams that are issued at periodic time intervals like MPEG video. Extended real-time Polling Service (ertPS): it requires low delay and data rate assurance like variable rate real-time data. Non-real-time Polling Service (nrtPS): In this services it requires minimum data rate and also support the RTNS delay tolerance like FTP, that is composed of variable size data packet and data streams. Lastly, Best Effort (BE): In this service, it doesn't require guaranteed of QoS for example, http. 
WiMAX network is the most preferred network adopted in tactical as well as cellular network [3] to provide the facility of data exchange and information sharing to enhance the process of military organization. For such network requires strict QoS provisioning and they usually interact with a static strategic network which has a larger bandwidth and more reliable communication links. Therefore, the efficient bandwidth allocation or scheduling mechanism is needed. In this allocation the bandwidth is dependent [4], on what the communication media can support/offer for cellular and tactical communication network.

The fundamental component of scheduling is a resource management in wireless network and also shows the main role in provisioning QoS such as delay, in different classes the data packet and throughput is losses in 802.16. This model i presented in [4], [5] isn't effective in using transmission capacity proficiently and can't foresee the number of users in network and also not able to predict time varying traffic of the network. Thus, the throughput efficiency and QoS of WiMAX network is affecting. To address in [6], [7] presented an evolutionary computing model to enhance QoS of WiMAX network by optimizing time and bandwidth requirement of subscriber. However, their approach is not efficient in synchronizing subscriber. Thus, incurs computation overhead. As a result, the real-time requirement of application may to be applicable. Therefore, synchronizing the subscriber and its application prerequisite is a significant parameter for efficient service provisioning.

In [8] presented a channel access mechanisms among subscriber using time slotted mechanism. Here, set of channel is shared among subscriber and each subscriber provides a dedicated slot time to transmit the packet. They presented a model namely throughput invariant i.e. it is time synchronized. However, improper measuring of synchronization will result in higher packet failure rate. Further, they did not considered service selectivity/prioritization requirement of varied service in WiMAX network. Along with it also induce bandwidth wastage since these model is implemented without feedback. To use bandwidth more efficiently and provision, number of methods has been presented in [9]-[12] with feedback in uplink scheduling. However, these model induces delay due to feedback computation. As a result, induce higher packet collision. Therefore, it is necessary to build a feedback based uplink scheduling mechanism that will give better QoS and reduce delay. For, attaining better QoS for uplink scheduling [7] presented a Meta heuristic particle swarm optimization (MHPSO) to compute time duration of frame that will offer better allocation and in [13] presented a genetic algorithm (GA) to optimize throughput of the network in the downlink scheduling. However, they did not consider optimizing uplink scheduling and it is not suitable for solving multi-objective problem.

In this work, we present an Adaptive Uplink Scheduling (AUS) for WiMAX network. This work presented an optimization of MAC scheduler for provisioning QoS. The MAC scheduler is use information from both physical layer and application layer. At Last, the application of QoS requirement bandwidth is use to efficiently this work presented an adaptive modulation selection scheme based on user application requirement using Multi-Objective Genetic Algorithm (MOGA).

The Contribution of research work is as follows:

a. The novelty of our work is we presented an application level based QoS provisioning for WiMAX network and multi-objective genetic algorithm is adopted to solve the optimization problem of QoS provisioning.

b. The overall result attained shows AUS model attain good performance in term of attaining higher throughput, successful packet transmission and minimizing packet collision.

The rest of the paper is organized as follows. In Section 2, the proposed adaptive uplink scheduling design for WiMAX is presented. In penultimate section experimental study is carried out. The conclusion and future work is described in last section.

\section{ADAPTIVE UPLINKSCHEDULING MODEL FOR WIMAX NETWORK}

This work present an Adaptive Uplink Scheduling (AUS) design for WiMAX network to provision QoS for both real-time and non-real rime traffic load and services. Firstly, we present architecture and system model and describes optimization problem of this work. Secondly, we present MAC scheduler optimization that use information from both physical layer and application layer. Lastly, to utilize bandwidth efficiently and meet QoS requirement of application this work presented an adaptive modulation selection scheme and multi-objective genetic algorithm (MOGA).

\subsection{Architecture of WiMAX Network}

This section describes the architecture of proposed WiMAX architecture. The architecture of this model is shown in Figure 1. WiMAX architecture is composed of Base station (BS) and set of subscriber station (SS) which is connected to base station. The base station is further connected with server or gateway. 
The connection among SS and BS is composed of uplink (UL) and downlink (DL). If the flow of traffic from SS is toward BS then it is called UL and if the flow of traffic from BS is toward SS then it is called DL.

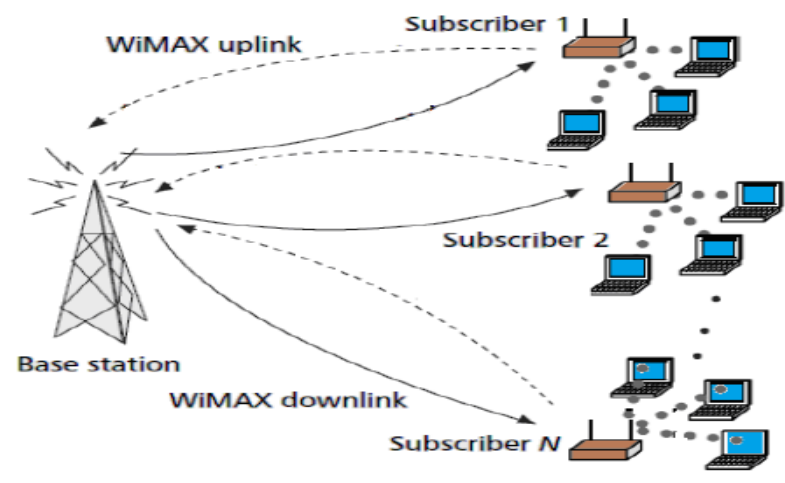

Figure 1. Architecture of WiMAX network

\subsection{System Model and Optimization Problem of WiMAX Network}

This section describes system model and optimization problem of our work. This paper considers WiMAX network composed of $O$ number of stations. The device pair $(j, k)$ represent the channel link among device $j$ and $k$ and all channel links is composed of a link set $M$. Let $s_{j k}$ depicts the channel link capacity of link $(j, k)$ and the channel link capacity is represented as follows:

$$
\vec{s}=\left[s_{j k},(j, k) \in M\right]
$$

Let consider that the overall channel capacity of a link is determined using interference from neighboring devices and transmitter transmission power. Therefore $\vec{s}$ can be expressed as follows:

$$
\vec{s}=v(\vec{Q})
$$

Where $\vec{Q}$ is the total channel link power assignment which can be expressed as follows:

$$
\vec{Q}=\left[Q_{j k},(j, k) \in M\right]
$$

Where $v$ is the rate power parameter, let us consider a traffic flow $t$ which is initialized from subscriber station (transmitter) to the base station (receiver) with a bandwidth is represented as follows:

$$
y_{t} \in\left[0, N_{t}\right]
$$

Where $N_{t}$ is the bandwidth limits. The bandwidth limit optimization problem for particular service is expressed as follows:

$$
\max _{y_{t \leq N_{t}}} \sum_{t} V_{t}\left(y_{t}\right)
$$

Such that $s_{j k}^{e} \geq 0 \forall(j, l) \in M$ and $\forall e \in E, E$ is the final set, where $V_{t}\left(y_{t}\right)$ is utility parameter that depicts the bandwidth utility. Based on different forms of $V_{t}$ (modulation coding rate) output throughput value is maximized. In this flow of rate balanced at the link node $j$, it requires the link capacity to be equal or more than input traffic. Considering these case we obtain

$$
\sum_{k:(j, k) \in M} s_{j k}^{e}-\sum_{k:(k, j) \in M} s_{k j}^{e}-\sum_{t: g_{t}=j, e_{t}=e} y_{t} \geq 0 \forall e \& \forall j \neq e
$$

Where $s_{j k}^{e}$ is capacity of channel $\operatorname{link}(j, k)$ destination for $e$.

In WiMAX network different services has different priority or selectivity to meet QoS requirement. For that a here we introduce a QoS function as follows: 


$$
V_{t}\left(y_{t}\right)=x_{t} \log \left(y_{t}\right)
$$

Where $\left(x_{t}\right)$ is the bias to provision each service traffic $t$ at device $j$. In WiMAX network, all load in initialized by subscriber station towards base station. Therefore the optimization problem is obtained as follows:

$$
\begin{aligned}
& \max _{y_{t} \leq N_{t}} \sum_{t} x_{t} \log \left(y_{t}\right) \\
& \text { Such that } s_{j c} \geq 0 \forall(j, c) \in T \\
& s_{j c}-\sum_{t: g_{t}=j, e_{t}=c} y_{t} \geq 0 \forall j \neq c
\end{aligned}
$$

Wherec is the base station (BS). Solving the optimization problem of above equation is done by using evolutionary approach. However, in WiMAX network, it is composed different selectivity or priority and modulation rates. Further to attain higher bandwidth they can changes their modulation based on received signal to noise ratio (SNR). Keeping the weight constant will affect the performance of network and violate the QoS requirement of application. This work present an adaptive modulation coding optimization approach and novel evolution approach using multi objective genetic algorithm is presented to overcome the challenges and problems.

\subsection{MAC Scheduling Optimization}

To attain enhanced performance, this work use feedback information from both physical and application layer which can be described as follows:

$$
x_{t}=\beta o\left(\varphi, Q_{0}\right)+(1-\beta) R_{t}
$$

where $\beta o\left(\varphi, Q_{0}\right)$ is the modulation used when packet failure probability (PFP) $Q_{0}$ and for a given signal to noise ratio $\varphi$ and $R_{t}$ is the QoS requirement parameter which depend on type of traffic or load used such as unsolicited grant services (UGS), Polling service (PS) and Best effort (BE). $\varphi$ is the bias parameter used for a chosen modulation scheme. An effective mechanism to solve optimization problem is to use genetic algorithm and adaptive modulation scheme which is presented in subsequent section.

\subsection{Adaptive Modulation Scheme}

To attain an efficient adaptive modulation selection method, the scheduling model should maximize the bandwidth under specific packet failure probability. Therefore, the objective of our design is to bring a good trade-off between attain superior bandwidth utilization and minimizing packet failure probability $Q_{0}$. Let consider that the transmission power to be constant and SNR is segmented into $N+1$ successive partitions, whose end points is depicted as $\left\{\varphi_{o}\right\}_{o=0}^{N+1}$, where $N$ is the amount of transmission type available. If the present signal to noise ratio $\varphi \in\left[\varphi_{o}, \varphi_{o+1}\right]$, for $0=1, \ldots, N$, type $o$ is chosen and respective modulation model is selected. When deep fading ensues, $\varphi$ fall into $\left[\varphi_{o}, \varphi_{1}\right]$, which is type 0 with no more thing to be sent. The end points of $\varphi_{o}$ is an important factor to be identified. This work consider adaptive white Gaussian channel. Therefore, the packet failure probability can be estimated or approximated considering $\varphi$ is greater than zero and less than $\varphi_{q o}$ as follows:

$$
\operatorname{PFP}_{o}(\varphi) \approx 1
$$

and if $\varphi$ is greater or equal to $\varphi_{q o}$ than PFP is approximated as follows:

$$
\operatorname{PFP}_{o}(\varphi) \approx \varphi_{q o} \exp \left(-g_{o} \varphi\right)
$$

By inversing the (10) and (11), the end point of signal to noise ratios can be computed if the end point packet failure probability of a service provider is $Q_{0}$ as follows:

$$
\begin{aligned}
& \varphi_{0}=1 \\
& \varphi_{o}=\frac{1}{h_{o}} \log \left(\frac{b_{o}}{Q_{0}}\right), o=1,2,3, \ldots, N \\
& \varphi_{N+1}=+\infty
\end{aligned}
$$

Using above equation a subscriber station can estimates its coding and modulation scheme using information of its present signal to noise ratio $\varphi$ from physical layer. 


\subsection{Multi-Objective Genetic Algorithm}

This section present a novel multi-objective genetic algorithm approach for attaining efficient scheduling design. Let consider that $\bar{Y}(u)$ is the chromosomes at each step $u$ which depicts the present bandwidth allocation. The two initial chromosomes are represented as follows:

$$
\begin{aligned}
& \bar{Y}_{1}(u)=\left\{y_{1}^{1}(u) \ldots y_{o}^{1}(u)\right\}, \\
& \bar{Y}_{2}(u)=\left\{y_{1}^{2}(u) \ldots y_{o}^{2}(u)\right\}
\end{aligned}
$$

The next two generation is represented as follows:

$$
\begin{aligned}
& \bar{Y}_{1}(u+1)=\left\{y_{1}^{1}(u+1) \ldots y_{o}^{1}(u+1)\right\}, \\
& \bar{Y}_{2}(u+1)=\left\{y_{1}^{2}(u+1) \ldots y_{o}^{2}(u+1)\right\} .
\end{aligned}
$$

Now obtain the QOS requirement from application layer to finalize $R_{t} \in S$, which computes the selectivity of this service classes. To define the adaptive modulation coding class of physical layer to finalize $o\left(\varphi, Q_{0}\right)$ for each subscriber considering present signal to noise ratio $\varphi$ and PFP. Now let's compute weight $x_{t}$ for each subscriber using (9). Employ MOGA to solve (8). The MOGA is composed of following phases such us Selection, Crossover, and Mutation. The MOGA iterates among all these phases till the desired fitness function is reached, which is described as follows

$$
f\left(\bar{Y}_{1}(u)\right)=\min _{y_{t} \leq N_{t}} \sum_{t}-x_{t} \log \left(y_{t}\right)
$$

The selection process is obtained as follows:

$$
q_{t}\left(y_{j}^{l}(u)\right)=\frac{\log \left(y_{j}^{l}(u)\right)}{\sum_{k=1}^{o} \log \left(y_{j}^{l}(u)\right)}
$$

Where $q_{t}\left(y_{j}^{l}(u)\right)$ is the selection probability of $y_{j}^{l}(u), l=1$ or 2 .

Similarly, the crossover process is obtained as follows:

$$
\begin{aligned}
& y_{j}^{1}(u+1)=\alpha y_{j}^{1}(u)+(1-\alpha) y_{j}^{2}(u) \\
& y_{j}^{2}(u+1)=\alpha y_{j}^{2}(u)+(1-\alpha) y_{j}^{1}(u)
\end{aligned}
$$

Where $\alpha$ is the crossover parameter. Then, in the mutation process, this work firstly construct arbitrary chromosome as follows:

$$
y_{j}^{l}(u+1) \in\left[\mathbb{U}_{\downarrow}, \mathbb{U}_{\uparrow}\right]
$$

Wherel $=1$ or $2, \mathbb{U}_{\uparrow}$ is the upper bound of the subscriber rate and $\mathbb{U}_{\downarrow}$ is the lower bound. Finally, bandwidth are allocated to different subscriber.

The proposed Adaptive Uplink Scheduling design using feedback information attain good performance when compared with state-of-art technique which is experimentally shown in next chapter.

\section{RESULT AND ANNALYSIS}

This section present experiment analysis of proposed Adaptive Uplink Scheduling (AUS) design for WiMAX performance attained over state of-art technique [8]. The experiment is conducted on Windows 10 64-bit operating system, Intel Pentium I-7 class 64-bit processer, 16 GB RAM, and dedicated 4 GB CUDA enabled NVIDIA GPU. The simulator is implemented using Dot Net framework 4.5 using C\# programming language, C++ library, and Visual studio 2017 platform. This work considers following WiMAX environment standard obtained from [14]. We used IEEE 802.16 network with point-to-multipoint architecture that composed of multiple subscriber station and a base station. The based station is connected (wired) through a server or gateway. Here we use Orthogonal Frequency Division Multiple Access (OFDMA) physical layer. More detail of physical layer parameter used can be obtained from Table 1. Further, experiment consider an urban environment setup to design long-term design of WiMAX wireless channel. For modelling path-loss this work used model presented in [15], for multipath fading effect we used 
model presented in [16]. Lastly, for interference modelling this work used neighboring cells in to consideration for computing packet failure probability and for shadowing modelling we considered lognormal random variable with zero mean and standard deviation of $8.9 \mathrm{~dB}$. We had used QPSK, QAM 16, and QAM64 modulation scheme with different coding rate. More information can be obtained from Table 1 . Based on Signal-to-noise ratio the modulation schemes changes dynamical. The complete simulation parameter used in this work are described in Table I. Experiment are conducted to evaluate performance of proposed AUS model over exiting model [8], namely TI (Throughput Invariant) sequence in terms of throughput, successful packet transmission and packet collision considering varied subscriber station, varied frequency slots, and varied time slots.

Table 1. Simulation Parameter Considered

\begin{tabular}{ll}
\hline Network Parameter & Value \\
\hline Network Size & $50 \mathrm{~m} * 50 \mathrm{~m}$ \\
Number of base station per region & 1 \\
Number of subscriber station & $20,30 \& 40$ \\
Modulation scheme & QPSK, QAM-16, QAM-64 \\
Coding rates & QPSK $(3 / 4)$, QAM-16 $(1 / 2,3 / 4)$, and QAM-64 $(2 / 3,3 / 4)$. \\
Number of Frequency Channels & 7 \\
Number of time slots & $8 \mu \mathrm{s}$ \\
Bandwidth & 9 to $27 \mathrm{Mbps}$ \\
Message information size & 27 bytes \\
\hline
\end{tabular}

\subsection{Throughput Performance Evaluation Considering Varied Subscriber Station, Varied Frequency Slots and Varied Time Slots}

This section present throughput performance attained by proposed AUS model over exiting TI sequence model considering varied subscriber station, frequency slots, and time slots. Figure 2 shows throughput performance attained by AUS model over TI sequence model considering varied subscriber station. The subscriber station are varied as 20,30, and 40 and AUS model attained a throughput improvement of $32.08 \%, 31.32 \%$, and $21.01 \%$ over TI sequence model respectively. An average throughput improvement of $28.14 \%$ is attained by proposed AUS model over TI sequence model. Further, this work conducted throughput performance evaluation considering varying frequency and time slot considering fixed number of subscriber station. Figure 3 shows the throughput performance outcome attained by AUC and TI sequence model for varied frequency considering 40 subscriber station. From Figure 3 it can be seen that throughput degrades with increase in frequency slots. The frequency slots is varied as 4, 6, and 8 and AUS model attained a throughput improvement of $22.85 \%, 22.1 \%$, and $14.64 \%$ over TI sequence model respectively. An average throughput improvement of $22.85 \%$ is attained by proposed AUS model over TI sequence model considering varied frequency slots. Similarly, Figure 4 shows the throughput performance outcome attained by AUC and TI sequence model considering varied time slots respectively considering 40 subscriber station. From Figure 4 it can be seen that throughput degrades with increase in time slots. The time slots is varied as 4, 6, and 8 and AUS model attained a throughput improvement of 16.06\%, 39.09\%, and $18.98 \%$ respectively. An average throughput improvement of $22.85 \%$ is attained by proposed AUS model over TI sequence model considering varied time slots. From overall result it can be seen as we increase subscriber station more number of packets are generated as result attain higher throughput. Further, as we increase the size of frequency and time slots, the throughput degrades since giving more time for packet transmission and permitting more user by dividing frequency result in bandwidth wastages and reduced data rate. An average throughput improvement of $24.24 \%$ is attained by proposed AUC over TI sequence model considering varied subscriber station, frequency slots, and time slots. The overall throughput result attained considering varied subscriber station, frequency slots, and time slots shows that AUC model is scalable and efficient. 


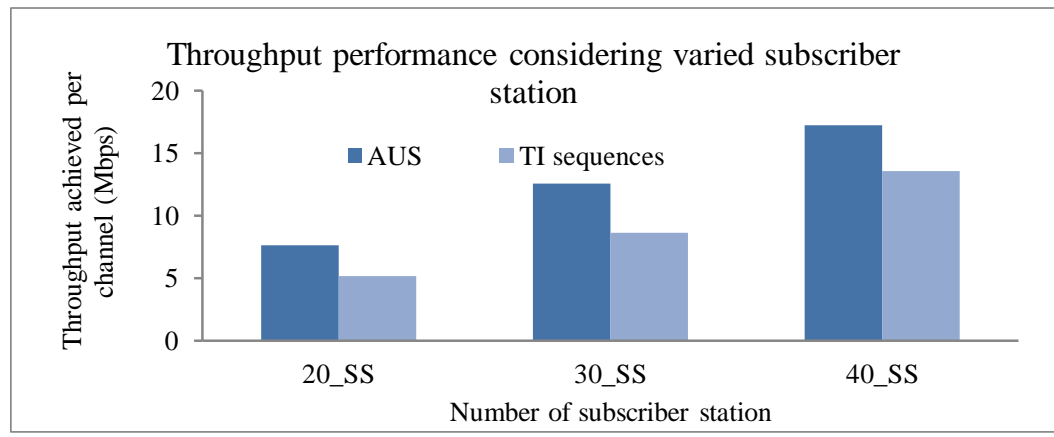

Figure 2. Throughput performance evaluation considering varied subscriber station

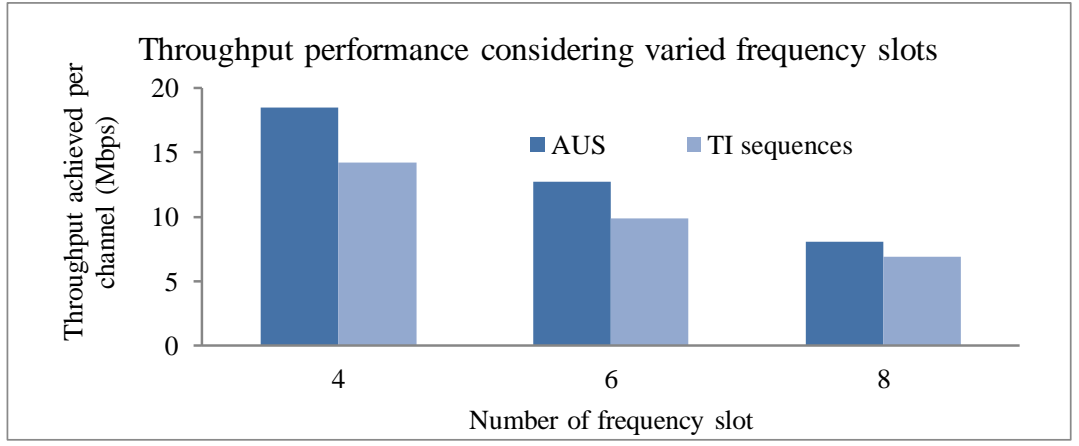

Figure 3. Throughput performance evaluation considering varied frequency slots

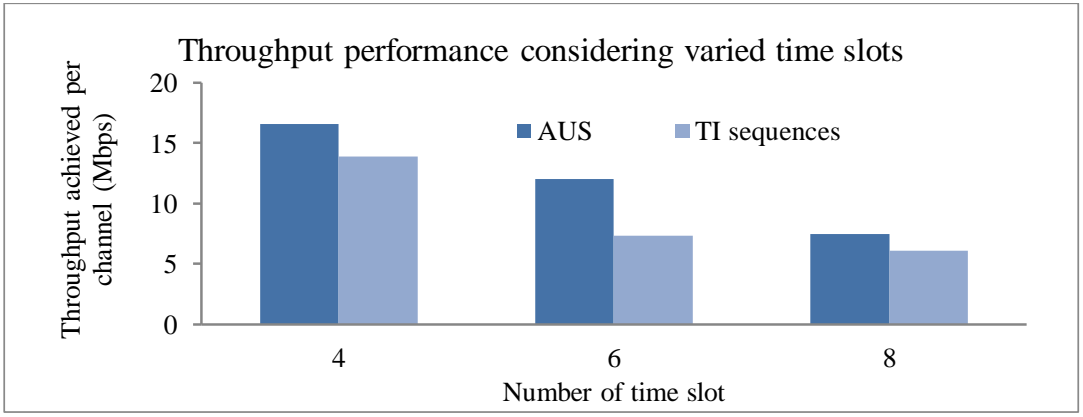

Figure 4. Throughput performance evaluation considering varied time slots

\subsection{Successful Packet Transmission Performance Evaluation Considering Varied Subscriber Station, Varied Frequency Slots and Varied Time Slots}

This section present successful packet transmission performance attained by proposed AUS model over exiting TI sequence model considering varied subscriber station, frequency slots, and time slots. Figure 5 shows successful packet transmission performance attained by AUS model over TI sequence model considering varied subscriber station. The subscriber station are varied as 20, 30, and 40 and AUS model attained a successful packet transmission improvement of $26.41 \%, 17.28 \%$, and $13.51 \%$ over TI sequence model respectively. An average successful packet transmission improvement of $19.07 \%$ is attained by proposed AUS model over TI sequence model. Further, this work conducted successful packet transmission performance evaluation considering varying frequency and time slot considering fixed number of subscriber station. Figure 6 shows the successful packet transmission performance outcome attained by AUC and TI sequence model for varied frequency considering 40 subscriber station. From Figure 6 it can be seen that successful packet transmission degrades with increase in frequency slots. The frequency slots is varied as 4 , 6 , and 8 and AUS model attained a successful packet transmission improvement of $27.52 \%, 35.44 \%$, and $23.53 \%$ over TI sequence model respectively. An average successful packet transmission improvement of $28.83 \%$ is attained by proposed AUS model over TI sequence model considering varied frequency slots.

Adaptive uplink schedulingmodel for WiMAX network using evolutionary computing model (Mahesh D S) 
Similarly, Figure 7 shows the successful packet transmission performance outcome attained by AUC and TI sequence model considering varied time slots respectively considering 40 subscriber station. From Figure 7 it can be seen that successful packet transmission degrades with increase in time slots. The time slots is varied as 4, 6, and 8 and AUS model attained a successful packet transmission improvement of 15.73\%, 25.92\%, and $34.042 \%$ respectively. An average successful packet transmission improvement of $25.23 \%$ is attained by proposed AUS model over TI sequence model considering varied time slots. From overall result it can be seen as we increase subscriber station more number of packets are generated as result attain higher successful packet transmission. Further, as we increase the size of frequency and time slots, the successful packet transmission degrades since giving more time for packet transmission and permitting more user by dividing frequency result in bandwidth wastages and reduced data rate. An average successful packet transmission improvement of $24.37 \%$ is attained by proposed AUC over TI sequence model considering varied subscriber station, frequency slots, and time slots. The overall successful packet transmission result attained considering varied subscriber station, frequency slots, and time slots shows that AUC model is scalable and efficient.

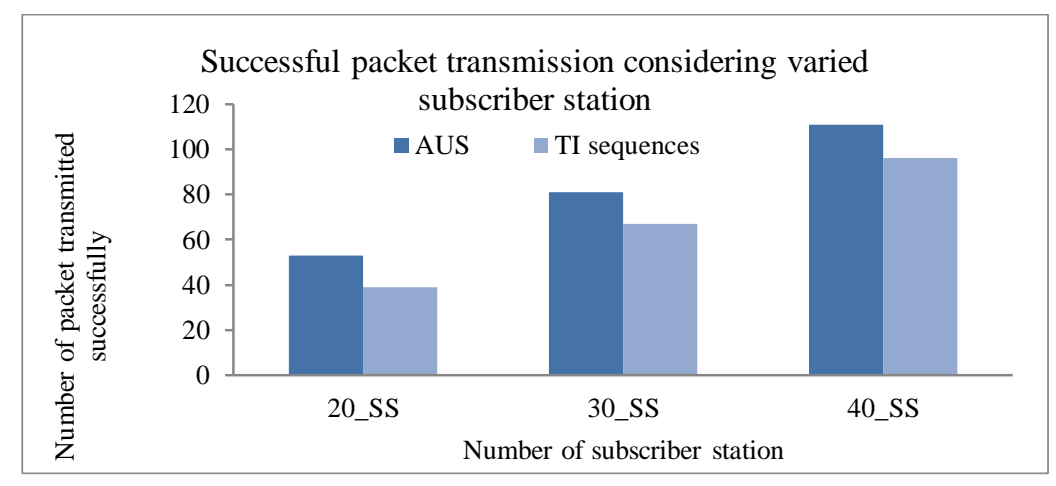

Figure 5. Successful packet transmission performance evaluation considering varied subscriber station

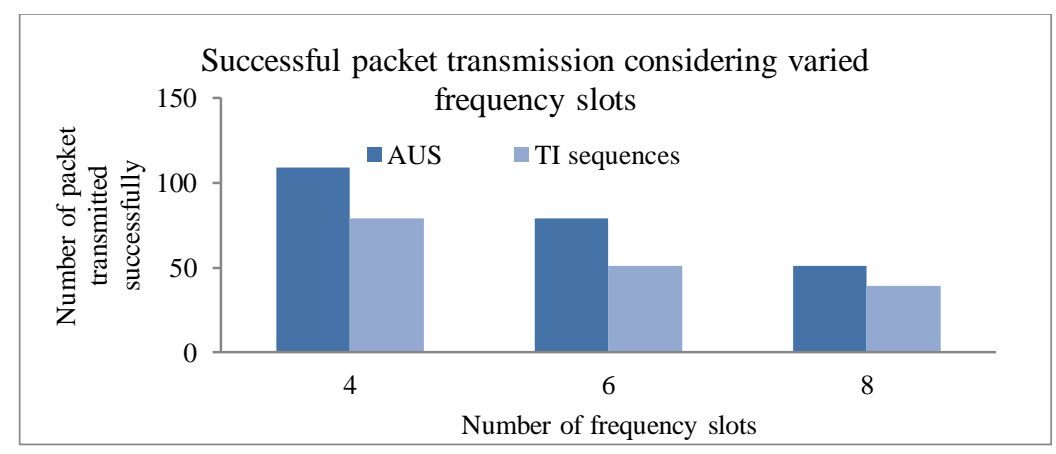

Figure 6. Successful packet transmission performance evaluation considering varied frequency slots

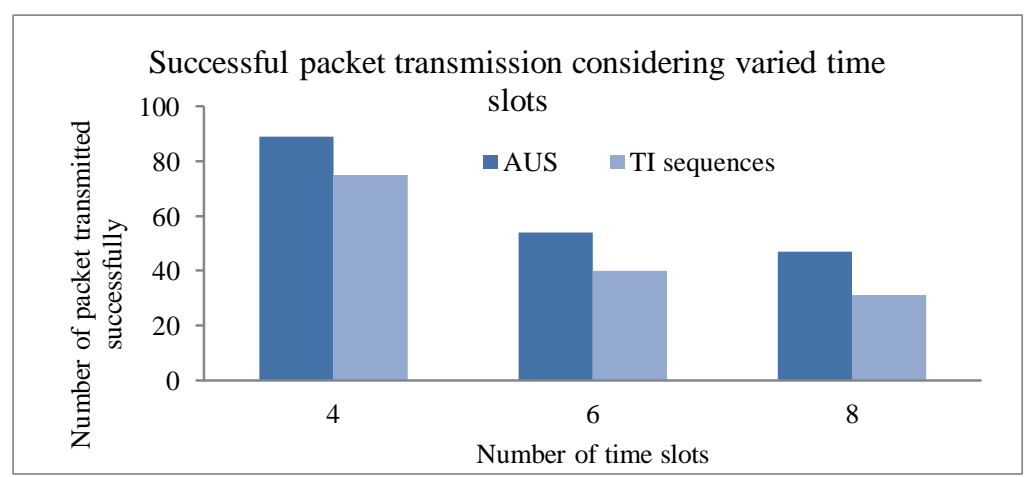

Figure 7. Successful packet transmission performance evaluation considering varied time slots 


\subsection{Collision Performance Evaluation Considering Varied Subscriber Station, Varied Frequency Slots and Varied Time Slots}

This section present collision performance attained by proposed AUS model over exiting TI sequence model considering varied subscriber station, frequency slots, and time slots. Figure 8 shows collision performance attained by AUS model over TI sequence model considering varied subscriber station. The subscriber station are varied as 20,30, and 40 and AUS model attained a collision reduction of $76.19 \%$, $36.73 \%$, and $11.67 \%$ over TI sequence model respectively. An average collision reduction of $41.53 \%$ is attained by proposed AUS model over TI sequence model. Further, this work conducted collision performance evaluation considering varying frequency and time slot considering fixed number of subscriber station. Figure 9 shows the collision performance outcome attained by AUC and TI sequence model for varied frequency considering 40 subscriber station. From Figure 9 it can be seen that less number of packet collision occurs with increase in frequency slots. The frequency slots is varied as 4, 6, and 8 and AUS model attained a collision reduction of $19.37 \%, 38.81 \%$, and $47.62 \%$ over TI sequence model respectively. An average collision reduction of $35.26 \%$ is attained by proposed AUS model over TI sequence model considering varied frequency slots. Similarly, Figure 10 shows the collision performance outcome attained by AUC and TI sequence model considering varied time slots respectively considering 40 subscriber station. From Figure 10 it can be seen that less number of packet collision occurs with increase in time slots. The time slots is varied as 4, 6, and 8 and AUS model attained a collision reduction of $13.01 \%, 36.71 \%$, and $62.16 \%$ respectively. An average throughput improvement of $37.29 \%$ is attained by proposed AUS model over TI sequence model considering varied time slots. From overall result it can be seen as we increase subscriber station more number of packets are generated as result attain higher collision. Further, as we increase the size of frequency and time slots, we can see that less number of packet collision occurs since giving more time for packet transmission and permitting more user by dividing frequency result in giving enough time and more slots for transmission to be successful. An average collision reduction of $38.03 \%$ is attained by proposed AUC over TI sequence model considering varied subscriber station, frequency slots, and time slots. The overall collision result attained considering varied subscriber station, frequency slots, and time slots shows that AUC model is scalable and efficient.

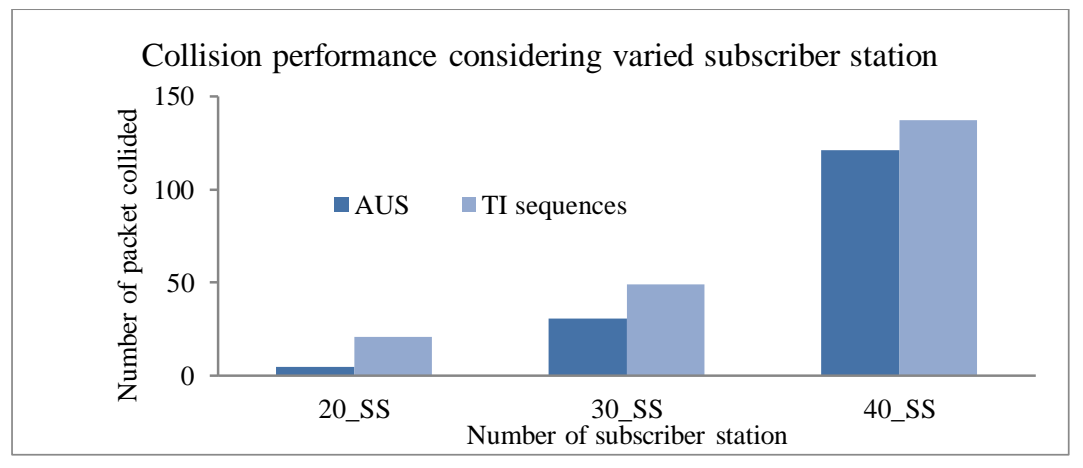

Figure 8. Collision performance evaluation considering varied subscriber station

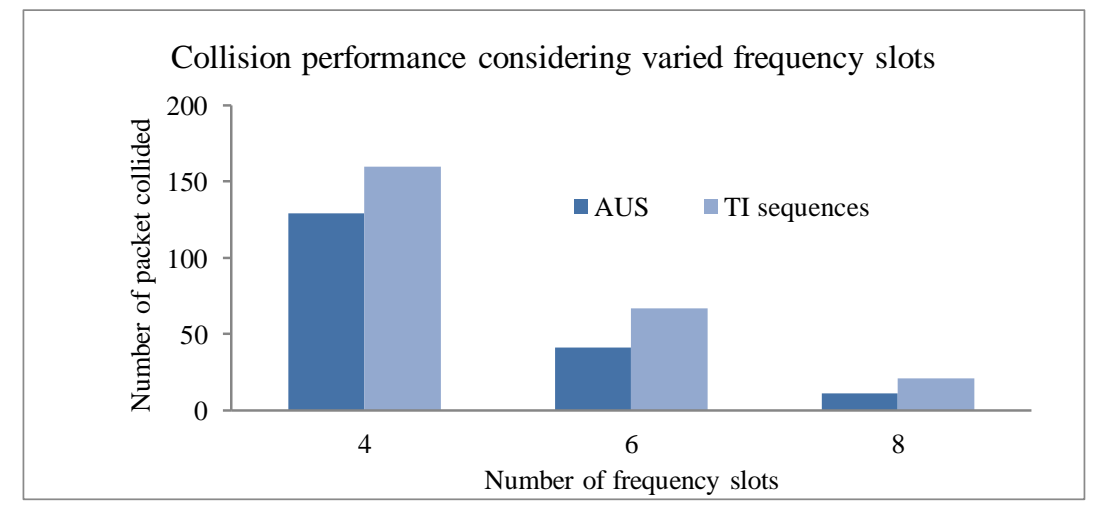

Figure 9. Collision performance evaluation considering varied frequency slots 


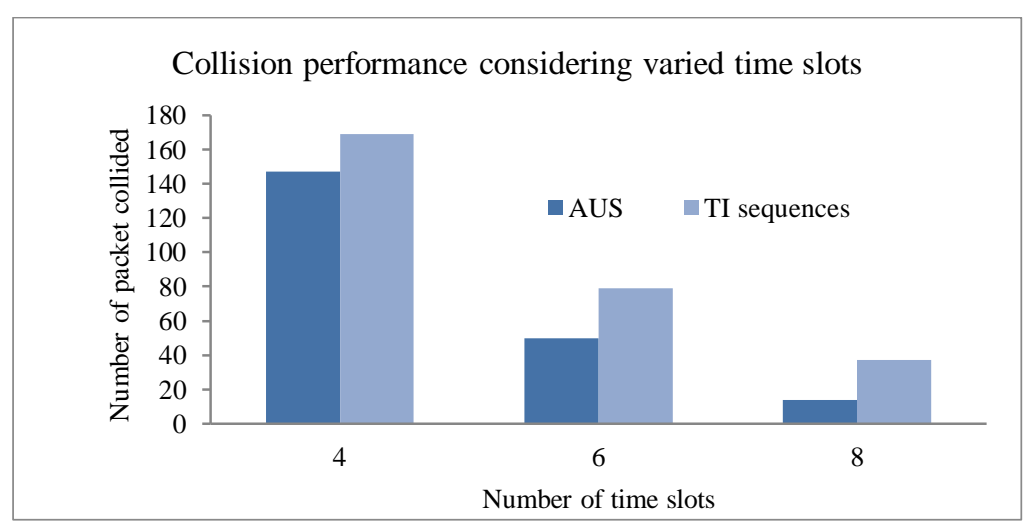

Figure 10. Collision performance evaluation considering varied time slots

\section{CONCLUSION}

This paper presented an efficient uplink scheduling model for WiMAX network. The model presented application level based QoS provisioning for WiMAX network. For attain higher bandwidth based on user application specific requirement the MAC scheduler changes modulation based on received signal to noise ratio. For, maximizing bandwidth utilization and user QoS requirement multi-objective, genetic algorithm is modeled. Experiment are conducted to evaluate performance of Adaptive Uplink Scheduling model over exiting model. The overall result attained shows AUS model attain good performance in term of throughput, successful packet transmission and packet collision. An average packet throughput performance improvement of $24.24 \%$ is attained by Adaptive Uplink Scheduling model over exiting model considering varied subscriber station and varied frequency slots and varied time slots. Further, an average successful packet transmission improvement of $24.37 \%$ is attained by Adaptive Uplink Scheduling model over exiting model considering varied subscriber stations and varied frequency slots and varied times slots. An average packet collision reduction of $38.03 \%$ is attained by Adaptive Uplink Scheduling model over exiting model considering varied subscriber station and varied frequency slots and varied time slots. The overall result attained shows scalable performance irrespective of subscriber station, frequency, time slots size.

In future we would further evaluate our proposed model considering varied network parameter. Further, we present a scheduling model to provision fairness for non-real time and polling service with minimum delay.

\section{REFERENCES}

[1] V. P. Firas Shawkat Hamid "The difference between IEEE 802.16/ WiMAX and IEEE 802.11 / Wi-Fi networks for Telemedicine Applications" IJRTE, ISSN: 2277-3878, Volume-2, Issue-5, 2013.

[2] M. Taghipoor, S. Mjafari and V. Hosseini, "Quality of Service and Resource Allocation in WiMAX," In Tech, Croatia, 2012.

[3] Anand R, Jitendranath Mungara," Wireless Environment Aware Adaptive Scheduling Technique for Cellular Networks", Indonesian Journal of Electrical Engineering and Computer Science Vol. 11, No. 1, July 2018, pp. 318 332.

[4] Avinash Kaur, Harvinder Singh, Parveen Sharma, "Bandwidth Allocation Scheduling Algorithms for IEEE 802.16 WiMax Protocol to Improve QoS: A Survey”, International Journal of Computer Applications (0975 - 8887) Volume 98- No.11, 2014.

[5] E. C. Rosa and P. R. Guardiero, "CAC and Uplink Scheduling Algorithms in WiMAX Networks," Telecommunications Magazine, vol. 13(2), pp. 32-39, ISSN 1516-2338, 2011.

[6] E. R. Dosciatti and A. Foronda, "Time frame optimization using PSO to guarantee QoS in IEEE 802.16 networks," Science and Information Conference (SAI), London, pp. 506-513, 2015.

[7] E. R. Dosciatti, W. Godoy Junior and A. Foronda, "TQ/PSO - A New Scheduler to Optimize the Time Frame with PSO in WiMAX Networks," in IEEE Latin America Transactions, vol. 13, no. 1, pp. 365-376, 2015.

[8] Y. Zhang, Y. H. Lo, W. S. Wong and F. Shu, "Protocol Sequences for the Multiple-Packet Reception Channel Without Feedback," in IEEE Transactions on Communications, vol. 64, no. 4, pp. 1687-1698, 2016.

[9] R. Amin and J. Martin, "Assessing Performance Gains Through Global Resource Control of Heterogeneous Wireless Networks," in IEEE Transactions on Mobile Computing, vol. 15, no. 2, pp. 292-305, 2016.

[10] M. Y. Arslan, K. Sundaresan, S. V. Krishnamurthy and S. Rangarajan, "iBUS: An Integrated Beamformer and Uplink Scheduler for OFDMA Small Cells," in IEEE/ACM Transactions on Networking, vol. 24, no. 2, pp. 901-914, 2016. 
[11] Raimi Dewan, M.K.A. Rahim, M.R. Hamid, M.F.M. Yusoff, H.A. Majid, B.A.F. Esmail, “ Dual Band to Wideband Pentagon-shaped Patch Antenna with Frequency Reconfigurability using EBGs" International Journal of Electrical and Computer Engineering (IJECE) Vol. 8, No. 4, August 2018, pp. 2557 2563.

[12] J. Yoon, M. Y. Arslan, K. Sundaresan, S. Krishnamurthy and S. Banerjee, "Characterization of Interference in OFDMA-based Small-cell Networks," in IEEE Transactions on Vehicular Technology.doi: 10.1109/TVT.2018.2839692, 2018.

[13] Prof. Dr. Gond V.J., Prof. Dr. Gond V.J, Prof. Dr. Abhay Wagh, Prof. Dr. Upena Dalal "Genetic Algorithm for Resource Allocation in WiMAX Network" International Journal of Computer Applications (0975 - 8887) Volume 35- No.2, December 2011.

[14] WiMAX Forum "Mobile WiMAX-Part I: A Technical Overviewand Performance Evaluation,” white paper, Aug. 2006.

[15] N. Blaunstein, Radio Propagation in Cellular Networks. ArtechHouse, 1999.

[16] ITU-R Task Group 8/1 "Guidelines for Evaluation of Radio Transmission Technologies for IMT-2000," Recommendation ITUR M.1225, 1999. 